\title{
Choosing Wisely campaign well received
}

$\mathrm{D}$ octors across the country are expressing wide support for the new Choosing Wisely Canada campaign, but whether the lists of unnecessary procedures will actually change practice remains to be seen.

The goal of the campaign is to change the culture of "more is better" in medicine, said founder Dr. Wendy Levinson at the Apr. 2 launch in Ottawa, Ontario.

So far, nine medical societies have published lists of the top five treatments, tests and procedures that are often unnecessarily performed in their respective fields, and 30 other groups are "at various stages of engagement in the campaign," according to the website. Several doctors suggested that the patient-education aspect of the campaign, including plain-language pamphlets, will make it easier to say no to unnecessary tests and treatments.

"The campaign is fantastic and a long time coming," says Dr. Daisy Fung, a family physician in Edmonton, Alberta. "I see myself using the website during the visit with the patient to facilitate the encounter."

But many of the recommendations cover well-trodden ground, calling into question why physician and patient education is required. That antibiotics shouldn't be used for influenza and viral sinusitis has been reiterated in the literature for more than a decade, for example.

"The extent to which I see these lists reflect well-established, long-ago agreed-upon recommendations is a bit disappointing to me," says Dr. Nancy Morden, an associate professor at the Dartmouth Institute for Health Policy and Clinical Practice in New Hampshire, and the author of a paper that critically analyzes the United States' Choosing Wisely campaign. "We're not advancing the identification of low-value care; we're just codifying it yet again."

Dr. Andrew Pinto, a family physician in Toronto, Ontario, and a health eco-

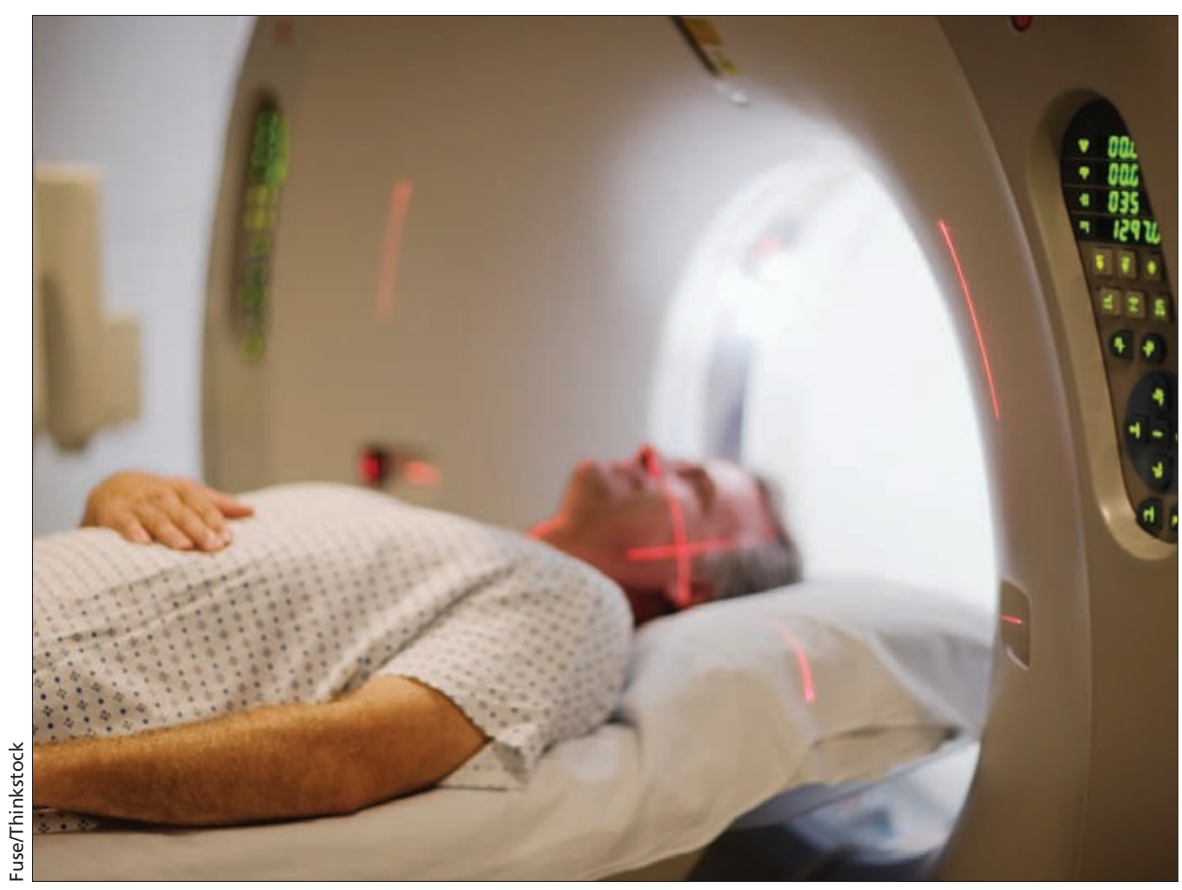

Avoiding medical imaging for minor head injuries in the absence of red flags is one recommendation in the Choosing Wisely Canada campaign.

nomics researcher at the Centre for Research on Inner City Health, argues that the lists will indeed have an impact, because they present information in a way that is "quite new" to medicine.

Although guidelines lay out "what you need to do," the campaign says "here are things you should avoid doing," Pinto explains.

Of course, outmoded practices continue in medicine not simply because of a lack of awareness, but also because of other factors, including patient demand and financial incentives.

"It takes me 30 seconds to write a prescription for antibiotics and it takes me 30 minutes to talk someone out of this prescription," says Morden. And, just as in the US, Canadian physicians aren't paid more to have longer patient consultations in primary care. "The incentives are misaligned to practise this kind of good care."

Dr. Jessica Otte, a family locum physician in Vancouver, British Columbia, recognizes that economics does come into play. Benzodiazepines and antipsychotics are often overprescribed to the elderly contrary to a Choosing Wisely recommendation - because it can be too difficult to deal with high numbers of patients becoming agitated or getting out of bed in the night, she explains.

"If we had more nurses, I think we'd use less of those," she says.

Still, she doesn't think regulation or financial disincentives are needed to discourage unnecessary procedures. (Otte is a member of the CMA's Health Care Transformation working group and blogs at www.lessismoremedicine .com, but wasn't involved in the Choosing Wisely campaign.)

She points to the 2012 regulation change in Ontario, which stated that imaging tests for low-back pain should be ordered only in cases of suspected or known pathology. "They probably saw it as a cost savings initiative ... and it seems to have changed practice, possibly for the better, but I think that's a really dangerous way to walk," she says.

The Choosing Wisely rules "will 
apply maybe $99 \%$ of the time, but there'll be that one case where you really wish you had access to the test," she says.

Dr. Rick Glazier, a family physician and senior scientist in primary care and population health at the Institute for Clinical Evaluative Sciences in Toronto, agrees that financial barriers or regulations could stand in the way of good medicine. Instead, he argues, it's necessary to "structure the environ- ment" to reflect the Choosing Wisely recommendations.

For example, preoperative checklists need to be rewritten so that electrocardiograms and chest X-rays aren't automatically called for; instead, providers ordering a preoperative test for a low-risk surgery patient should be required to "state the clinical reason for doing so."

Such structural changes are likely a next step, says Glazier. "There are two aspects of [the campaign] that are really different and have great potential." The first is that it's targeted to the public, and the second is that it's driven not only by scientists and medical journals, but by the professional organizations themselves. "For radiologists to come forward and say you shouldn't order radiology tests under these circumstances, this is unprecedented." Wendy Glauser, Toronto, Ont.

CMAJ 2014. DOI:10.1503/cmaj.109-4779 